\title{
VALENCE ET RELATIONS GRAMMATICALES
}

\section{La notion de valence}

Dans la théorie de la valence, telle qu'elle a d'abord été conçue par Tesnière (1959), le verbe constitue le centre organisateur de la phrase. Les spécifications lexicales d'un verbe donné sont traduites par ses possibilités combinatoires, c'est-à-dire par les membres nominaux et/ou prépositionnels avec lesquels il se combine: ${ }^{1}$ toute compréhension d'un verbe tel que envoyer, par exemple, prend son point de départ dans le fait que ce verbe dénote une situation comportant trois participants qui sont dénotés par les trois membres de phrase avec lesquels se combine ce verbe. De ce fait, la notion de valence n'offre pas seulement une façon simple de classifier les verbes d'une langue donnée, mais aussi une hypothèse sur la structure des phrases de la langue: si c'est le verbe qui détermine la structure de la phrase, la théorie de la valence doit être construite de telle sorte qu'elle permette l'élaboration d'une typologie de phrases en même temps qu'une analyse et une classification des verbes. Ce qu'il faut exiger d'une théorie de la valence, c'est donc qu'elle formule une hypothèse sur la structure des phrases - les schémas canoniques - c'est-à-dire qu'elle prédise quels types de phrase existent et quels types sont exclus par principe. On n'atteint pas ce but si on se contente de l'inventaire flou et aléatoire des fonctions syntaxiques de la grammaire traditionnelle pour en faire un principe de classification des verbes: avec un tel point de départ, on formulera en réalité l'hypothèse qu'il existe des phrases comportant jusqu'à sept ou huit actants, et on se trouvera devant des lacunes distributionnelles inexplicables comme par exemple l'absence d'une fonction "attribut de l'objet indirect", lacune d'autant plus mystérieuse qu'on dit le plus souvent que l'objet indirect n'est qu'une variante prépositionnelle de l'objet direct, qui, lui, a son attribut.

J'essaierai de montrer qu'une conception restrictive de la notion de valence pourra comporter des hypothèses intéressantes sur la structure des phrases en français. Mon point de départ est que la valence se définit en termes de relations grammaticales et non pas en termes, par exemple, de configurations syntactico-lexicales (NP, PP, etc.). Je

1 Tesnière lui-même formule quelques réserves en ce qui concerne le statut des syntagmes prépositionnels, mais ces réserves semblent plutôt relever de contradictions internes dans sa théorie. Voir la discussion dans Herslund (1988a:10-13). 
suppose, tout simplement, que les verbes, parmi leurs spécifications lexicales, comportent des références à des relations telles que sujet, objet, etc. Ces relations sont les expressions syntaxiques de relations lexicales. La valence d'un verbe donné est donc définie par les relations auxquelles réfêrent les spécifications lexicales de ce verbe.

\section{Les actants}

Je suppose, dans ce qui suit, qu'il existe une distinction pertinente entre les membres de phrase qui sont liés, c'est-à-dire déterminés par le contenu lexical du verbe et les membres de phrase qui sont libres dans le sens qu'ils ne sont pas prévus par le contenu lexical du verbe, et qui sont, parfois, ajoutés à la phrase entière. Je suppose de plus que cette distinction peut être établie et justifiée dans tous les cas par des critères précis et opérationnels (comme on le sait, tel n'est pas le cas, cf. par exemple Vater 1978). Je distinguerai en outre entre arguments et différents types de modifieurs adverbiaux. On obtient ainsi une quadripartition des membres de phrase:

(1)

\begin{tabular}{l|l|l|} 
& lié & libre \\
\hline argument & actant & circonstant \\
\hline modifieur & adverbe lié & adverbe libre \\
\hline
\end{tabular}

Seuls les actants définissent la valence à proprement parler. Les exemples de (2) illustrent différents membres de phrase liés, arguments et modifieurs:

(2) a. Cela ennuie le colonel.

Le marchand pèse les légumes.

Julie lit son livre favori.

b. Cela coûte $\mathbf{1 2 0}$ francs.

Ce sac pèse 13 kilos.

Julie se comporte bien.

Les syntagmes en caractères gras de (2) b. dépendent clairement du verbe, mais ne semblent pas constituer les arguments d'une relation. Or, le propre d'un verbe est de dénoter une relation. Les syntagmes en caractères gras sont plutôt des quantifieurs ou des modifieurs de l'état ou de l'activité dénotés par le verbe: c'est la relation elle-même qui est modifiée ou quantifiée par ces syntagmes, ce n'est pas une relation qui est établie. ${ }^{2}$ Seuls les membres de phrase qui correspondent à des arguments d'une relation auront le statut d'actants. Ces membres définissent seuls la valence du verbe.

2 La grammaire normative du français écrit distingue les compléments comme ceux de (2) b. de l'objet direct par l'absence d'accord du participe passé: Les deux cents francs que cette valise m'a coûté. 


\section{Les relations grammaticales}

Comme je l'ai avancé ci-dessus, la tâche principale d'une théorie de la valence consiste à établir un inventaire de relations grammaticales qui permettent de prédire les types de phrases existants à partir d'une classification des verbes aussi simple que possible. Il faut donc se demander quelles relations les actants contractent avec le verbe et, éventuellement, entre eux. Autrement dit, quelles relations grammaticales un verbe peut-il spécifier?

$\mathrm{Si}$ les verbes dénotent des relations, les termes de ces relations, les arguments, sont caractérisés par des rôles sémantiques. Comme il existe autant de rôles que de verbes différents, le rôle de la syntaxe semble justement être celui de généraliser ces rôles et de les convertir en configurations syntaxiques autour du verbe. Cette réduction et conversion a lieu en deux étapes selon le rôle que jouent les arguments par rapport à deux entités primitives: le prédicat et la proposition.

Le principal argument est celui avec lequel le verbe forme l'unité que j'appelle un prédicat syntaxique. C'est l'argument qui dénote l'entité sans laquelle la situation dénotée par le verbe ne peut être comprise ou même conçue: cet argument est l'actant fondamental $\mathrm{du}$ verbe. Cette relation fondamentale est illustrée par un verbe symétrique comme casser:

(3) a. Le fil casse.

b. Julie casse le fil.

Dans ces phrases, l'actant fondamental, qui dénote "l'entité qui se casse", est exprimé comme sujet d'une phrase intransitive et comme objet d'une phrase transitive.

L'autre argument essentiel est celui sans lequel il n'y aurait pas de proposition. Autrement dit, l'argument qui s'unit au prédicat syntaxique de sorte qu'une séquence de mots est perçue comme constituant une proposition, c'est-à-dire une unité de sens à laquelle on peut attribuer une valeur de vérité. Appelons cette opération nexus ou prédication. Nous pouvons alors dire que l'actant fondamental se combine avec le verbe pour former avec lui un prédicat syntaxique, alors que l'autre argument se combine avec ce prédicat pour former une proposition:

(4) Phrase intransitive:

\section{Phrase transitive:}

$$
\underbrace{\left[\begin{array}{c}
\mathrm{y}+\mathrm{V}] \\
\text { prédicat }
\end{array}\right.}_{\text {proposition }}
$$

$$
\underbrace{\mathrm{x} \underbrace{[\mathrm{V}+\mathrm{y}]}_{\text {prédicat }}}_{\text {proposition }}
$$

Les efforts que ce travail lui a coûtés.

Les cent kilos qu'il a pesé autrefois.

Les trois kilos de pommes que le marchand a pesés.

Exemples provenant du Robert Méthodique. 
Comme il ressort de (3) et de (4), l'actant fondamental est l'objet d'un verbe transitif $(\mathrm{O})$ ou le sujet d'un verbe intransitif $\left(\mathrm{S}_{\mathrm{i}}\right)$, alors que l'actant qui constitue la proposition est le sujet $(\mathrm{S})$. Les différentes fonctions des actants dans la constitution de la phrase ressortent du schéma suivant:

\begin{tabular}{llll} 
prédicat & + & $S_{i}$ & $S_{t}$ \\
proposition & - & + & - \\
\hline
\end{tabular}

Il ressort de ce schéma que l'actant fondamental - l'argument qui constitue le prédicat syntaxique - peut être le même que celui qui constitue la proposition. C'est le cas du sujet intransitif, qui joue un rôle double dans la constitution de la phrase: il assure les deux fonctions qui, dans la phrase transitive, sont réparties sur $\mathrm{O}$ et $\mathrm{S}_{\mathrm{t}}{ }^{3} \mathrm{Il}$ y a ainsi une équivalence profonde et essentielle entre $S_{i}$ et $O$ : les deux relations partagent la propriété d'être constitutives du prédicat syntaxique. Cette identité profonde entre les deux relations est révélée par beaucoup de propriétés sémantiques et syntaxiques, par exemple par des restrictions de sélection lexicale communes à $S_{\mathrm{i}}$ et $\mathrm{O}$ (voir par exemple Herslund 1988a, 1988b).

Le fait que l'actant fondamental, $S_{i}$ ou $O$, par la création de l'unité appelée "prédicat syntaxique", soit plus étroitement lié au verbe que le sujet transitif, donc qu'il soit vraiment "fondamental", ressort clairement du fait que le rôle sémantique assigné à $S_{t}$ est déterminé par la combinaison verbe $+O$. En faisant varier le $O$ d'un verbe transitif, on fait varier du même coup le rôle du $S_{t}$ (cf. Marantz 1984:23 ss.):

(6) Julie a pris son manteau.

Julie a pris une aspirine.

Julie a pris une décision.

Julie a pris rendez-vous chez le dentiste.

Julie a pris le train.

Julie a pris son courage à deux mains.

C'est cette unité, le prédicat syntaxique, verbe $+O$, qui détermine le rôle sémantique du $S_{t}$, d'où les différentes lectures de prendre dans ces exemples: le rôle sémantique de $S_{t}$ varie en fonction du $O$, alors qu'on pourra faire varier par exemple le sujet de a pris le train à l'infini sans obtenir le moindre changement dans le prédicat.

On peut montrer à peu près la même chose pour le $\mathrm{S}_{\mathrm{i}}$ : comme le $\mathrm{O}$, il découpe et précise la polysémie inhérente à tout verbe, dont le sens varie par conséquent en fonction du sujet choisi. Voici des exemples de cette variation:

(7) Le train sort du tunnel.

Le clou sort du mur.

Ce journal sort tous les jours. 


\section{Julie sort souvent le samedi. ${ }^{4}$}

Avec les deux types d'actants identifiés maintenant - l'actant fondamental, $S_{i}$ ou $O$, et l'actant qui constitue la proposition, $S_{i}$ ou $S_{t}-$ on pourrait dire qu'il n'y a plus de place autour du verbe. Il n'y a en effet qu'une situation où le verbe peut spécifier un troisième actant: c'est la possibilité qu'ont certains verbes d'établir une relation de nature prédicative entre un de leurs actants et ce troisième actant. C'est la relation que j' appellerai adjet (A). ${ }^{5}$ Cette relation remplace un grand nombre de fonctions assez hétérogènes de la grammaire traditionnelle: l'objet indirect, l'objet prépositionnel, le complément local, le complément d'attribution, l'attribut du sujet et l'attribut de l'objet. $\mathrm{Ce}$ que toutes ces fonctions ont en commun, c'est le fait qu'elles expriment non seulement une relation au verbe, mais en même temps une relation à un autre actant, à savoir à l'actant fondamental, jamais au $\mathrm{S}_{\mathrm{t}}$. Cette conception d'une relation syntaxique double est évidemment contraire à un dogme fondamental de la théorie valentielle traditionnelle, cf. Happ (1978:108 s.), à savoir que les relations grammaticales relient uniquement le verbe à ses actants, jamais ces derniers, entre eux. Ce phénomène, une relation syntaxique double, établie par le verbe à la fois entre celui-ci et un actant, et entre cet actant et l'actant fondamental du verbe, est pourtant traditionnellement reconnu dans le cas des attributs (du sujet et de l'objet):

(8) a. François est président.

b. Ils ont élu François président.

Ajoutons qu'en accord avec les prédictions de la théorie avancée ici, l'attribut du sujet est toujours relié à un $S_{\mathrm{i}}$, jamais à un $\mathrm{S}_{\mathrm{t}}$ : il n'existe tout simplement pas de verbe transitif qui se fait suivre par un attribut du sujet, possibilité que n'exclut pas la grammaire traditionnelle, pourtant; mais une théorie de la valence doit expliquer ce fait. Nous retrouvons ici l'identité profonde entre $S_{i}$ et $O$ signalée plus haut: seulement l'actant fondamental peut être relié à un A. Dans (8), le syntagme président manifeste la

En comparant ces faits, qui illustrent le comportement des verbes inaccusatifs, à l'autre classe de verbes intransitifs, les inergatifs, on s'aperçoit que leur sujet se comporte plutôt comme le sujet transitif:

Julie danse.

Les feuilles mortes dansent.

Des images dansent sur le mur.

Le sens de danser ne varie pas; tout au plus peut-on observer différents degrés de lecture métaphorique des sujets. Des faits comme ceux-ci étayent une analyse selon laquelle les verbes inergatifs sont en réalité des verbes transitifs, voir Herslund (à paraître).

5 Cette relation était d'abord appelée objet indirect (cf. Herslund 1988a et b). Comme ce terme peut prêter à confusion, je préfère maintenant le terme adjet. Ce terme a au moins trois avantages: il est nouveau, il n'a donc pas de sens technique dans aucune théorie; il est formé, parallèlement à sujet et objet, selon les règles de formation de mots en latin avec des éléments latins; et dans la mesure où le terme évoque des associations, il fait penser au terme adjectif, ce qui n'est pas une association fâcheuse puisque un des traits fondamentaux de la relation est justement sa nature prédicative, ou "adjectivale", si on veut. Le terme est utilisé pour la première fois dans Sørensen (1988) 
relation $\mathrm{A}$, qui, dépendant du verbe, relie aussi ce syntagme à $S_{i}$ en a., à $O$ en $b$. Les verbes être et élire sélectionnent un $\mathrm{A}$ et introduisent par là une nouvelle relation dans la phrase, relation qu'on peut qualifier de prédicative:

(8') Phrase intransitive:<smiles>[AlH2]C=C[AlH2]</smiles>

préd. second.

\section{Phrase transitive:}

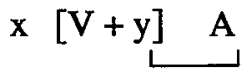

préd. second.

La reconnaissance d'une relation prédicative secondaire dans le cas des attributs ne constitue pas de nouveauté, mais je pense qu'il y a de bonnes raisons pour admettre une relation de même nature dans le cas des autres manifestations de $\mathrm{A}$ aussi, à savoir le complément local, l'objet prépositionnel et le complément datif.

Je limite ici la discussion aux compléments actantiels introduits par la préposition $\grave{a}$, qui constituent la classe la plus importante de "compléments indirects" en français. Je suppose que la base sémantique commune à tous ces compléments est de nature locative. ${ }^{6}$ Commençons donc notre discussion par le complément local, qui représente cette base assez directement:

(9) a. Julie est arrivée à Nice.

b. J'ai envoyé Julie à Nice.

Comme on le voit, le verbe arriver dénote l'établissement d'une relation locale entre son $\mathrm{S}_{\mathrm{i}}$ et son $\mathrm{A}$ - à vrai dire, entre les entités dénotées par les syntagmes qui sont les arguments de ces relations, Julie et à Nice - et le verbe envoyer entre son O et son A. Rappelons qu'aucun verbe n'établit de relation semblable entre son $S_{t}$ et son A: la relation est toujours et uniquement une relation entre l'actant fondamental et le A. Syntaxiquement parlant, on peut dire que arriver et envoyer établissent des relations de type prédicatif entre leur actant fondamental et leur A (cf. (8')). Et on peut dire qu'une partie du sens des phrases de (9) est rendue par la paraphrase (10), qui correspond à la prédication secondaire introduite par la relation A:

(10) 'Julie être à Nice'

Or, un verbe comme envoyer peut aussi être suivi d'un complément datif:

Dans le cas des attributs, cette interprétation n'est peut-être pas toujours tout à fait évidente. Mais je pense qu'il y a de bonnes raisons pour l'adopter. D'abord, la limite entre attribut et complément local n'est pas toujours très tranchée, $\mathrm{cf}$. la série suivante:

Il est à la prison de Fresnes.

Il est en prison.

Il est en colère.

Il est maussade.

Ensuite, l'interprétation locative d'exemples tels que (8) peut être explicitée par une paraphrase comme:

'François est placé dans la classe des présidents'

Pour un traitement formel de ces questions, voir Sørensen (1993). 
(11) J'ai envoyé un cadeau à Julie.

Si à Nice de (9) correspond à une structure pronominale avec le pronom $y$, c'est au pronom lui que correspond à Julie de (11); il y a d'autres différences aussi: la préposition est toujours à avec le complément datif, mais elle varie en fonction du syntagme régime dans le complément local (ces détails ne seront pas discutés ici, voir Herslund 1988a:36 ss.). Le même verbe permet donc deux manifestations différentes de son A. Une raison immédiate pour dire qu'il s'agit de deux manifestations de la même relation, et non pas de deux relations différentes, est donc que ces deux compléments s'excluent mutuellement: on ne peut pas avoir en même temps un complément local (actant!) et un complément datif. Nous pouvons alors dire que envoyer choisit ou bien un adjet locatif $\left(A_{l o c}\right)$ ou bien un adjet datif $\left(A_{d a t}\right)$. La même chose se vérifie pour beaucoup de verbes transitifs (pour des listes de ces verbes, voir Herslund 1988a).

Quelle est la différence entre ces deux manifestations de la même relation? Si on peut proposer une paraphrase de la prédication secondaire de (11) qui ressemble à (10), à savoir (12):

(12) 'un cadeau être à Julie'

on peut penser qu'une paraphrase plus adéquate serait plutôt quelque chose comme (12'):

(12') 'Julie avoir un cadeau'

Autrement dit, le datif fait figure d'un locatif renversé: alors que c'est le $O$ qui est sujet de la prédication secondaire dans le schéma $\mathbf{O} \mathbf{A}_{\text {loc, }}$ c'est le A qui constitue le sujet secondaire dans le schéma $\mathbf{O}$ Adat. Cette hypothèse permet en effet un grand nombre d'observations et a beaucoup de conséquences que je dois renoncer à poursuivre ici (voir Herslund 1988a pour les détails de la question). Mais la conclusion générale est que les fonctions syntaxiques de la grammaire traditionnelle énumérées ci-dessus manifestent en réalité la même relation, l'adjet.

Comme on l'a déjà vu, ces manifestations s'excluent mutuellement. C'est pourtant souvent le cas - en fait, c'est pratiquement la situation normale - qu' un verbe donné peut sélectionner plusieurs d'entre elles, mais évidemment jamais plus d'une à la fois, cf. par exemple le verbe mettre:

(13) a. Il a mis les bouteilles sur la table. $\left(\mathrm{A}_{\text {loc }}\right)$

b. Il lui a mis ses nouvelles chaussures. $\left(\mathrm{A}_{\text {dat }}\right)$

c. Il l'a mis de mauvaise humeur. $\left(A_{\text {Attro }}\right)$

Cette interprétation permet aussi de répondre à la question initiale: pourquoi n'a-t-on jamais signalé l'existence d'un attribut de l'objet indirect? Il découle de l'hypothèse présentée ici qu'une telle relation ne pourrait pas exister pour la simple raison qu'objet indirect et attribut sont des manifestations de la même relation, A, et parce qu'on ne peut pas avoir plus d'une manifestation de la même relation à la fois. On a du même coup une explication du fait qu'on ne trouve jamais de verbe qui se construit 
à la fois avec un complément local et un complément datif, mais beaucoup de verbes qui se construisent tantôt avec l'un, tantôt avec l'autre.

\section{Conclusion: relations grammaticales et structure de la phrase}

Avec l'hypothèse des trois types d'actants discutée ci-dessus, $S\left(S_{i}\right.$ et $\left.S_{t}\right), O$ et $A$, et la distinction fondamentale entre verbes transitifs et intransitifs qui en découle, on peut établir quatre classes de verbes. Si on y ajoute la classe des symétriques, les verbes à la fois transitifs et intransitifs, tels que casser, cf. par exemple Rothemberg (1974), on obtient en tout six classes de verbes:

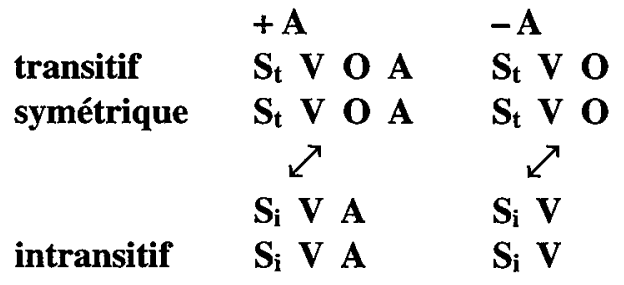

Ces six classes permettent de formuler l'hypothèse de la structure des phrases qu'on doit exiger d'une théorie de la valence. Mon hypothèse est que les phrases du français, et de toutes les langues du même type, se laissent décrire en termes des quatre schémas canoniques qu'on peut dériver des six classes de verbes:
(15) Phrase transitive
Phrase intransitive
$S$ V O A S V O
S V A S V

Les structures dérivées, causatif, passif, moyen, etc., peuvent être décrites comme des "dérivations" à l'intérieur des limites que définit la classification, c'est-à-dire que ces dérivations ne produisent pas de nouveaux types de phrases, voir par exemple Herslund 1988 b.

\section{Bibliographie}

Happ, Heinz (1978) "Théorie de la valence et enseignement du français". Le français moderne 46.97-134.

Herslund, Michael (1988a) Le datif en français. Editions Peeters, Louvain-Paris.

Herslund, Michael (1988b) "On Valence and Grammatical Relations". In F. Sørensen, éd.: Valency. Three Studies on the Linking Power of Verbs, p. 3-34. Copenhagen Studies in Language, CEBAL Series 11.

Herslund, Michael (1990) "Les verbes inaccusatifs comme problème lexicographique". Cahiers de Lexicologie 56-57.35-44.

Herslund, Michael (à paraître) "Les verbes intransitifs". 
Marantz, Alec (1984) On the Nature of Grammatical Relations. The MIT Press, Cambridge, Mass.

Olié, Annie (1984) "L'hypothèse de l'inaccusatif en français". Lingvistica Investigationes 8.363-401.

Rothemberg, Mira (1974) Les verbes à la fois transitifs et intransitifs en français. Mouton, La Haye.

Sørensen, Finn (1988) "Indice d'infinitif ou préposition. Comment intégrer cette distinction dans un analyseur". In Herslund et alii, éds.: Traditions et tendances nouvelles des études romanes au Danemark, p. 223-233. Etudes Romanes de l'Université de Copenhague 31. Munksgaard, Copenhague.

Sørensen, Finn (1993) "The Adject Constraint". The NORDLEX Project. Lexical Studies in the Scandinavian Languages. LAMBDA 18.63-73. Ecole des Hautes Etudes Commerciales de Copenhague.

Tesnière, Lucien (1959) Eléments de syntaxe structurale. Klincksieck, Paris.

Vater, Heinz (1978) "On the Possibility of Distinguishing between Complements and Adjuncts". In: W. Abraham, éd.: Valence, Semantic Case and Grammatical Relations, p. 21-45. John Benjamins, Amsterdam.

\section{Povzetek \\ VEZLJIVOST IN SLOVNIČNA RAZMERJA}

Če je glagol organizacijsko jedro stavka, potem glagolska vezljivost, ki pogojuje klasifikacijo glagolov, opredeljuje tudi različne tipe stavkov. Prispevek predstavlja skladenjski opis, narejen na osnovi pojma vezljivosti. Upošteva samo tri slovnična delovalniška razmerja: med osebkom, predmetom in nepremim predmetom. Prispevek analizira ta tri razmerja v francoščini. Opis izhaja iz pregleda francoskih glagolov ter pripelje do njihove razvrstitve $v$ šest skladenjskih razredov ter do tipologije vseh možnih stavkov $v$ francošcini (4 vrste). 